\title{
Products of Incomplete gamma functions Integral representations
}

\author{
Rami AlAhmad
}

(Communicated by İ. Onur KIYMAZ)

\begin{abstract}
In this paper we find integral representations, involving incomplete gamma and incomplete beta functions, of products of incomplete gamma functions. Also, in this paper we find interesting relations between incomplete gamma functions and Laplace transform. Since the error function is an incomplete gamma function, we find interesting relations between error functions and Laplace transform. Using the results above we find several interesting integrals.
\end{abstract}

Keywords: Incomplete gamma functions, Incomplete beta functions, error functions, Laplace transform.

AMS Subject Classification (2010): Primary: 33B20 ; Secondary: 44A10.

\section{Introduction}

Definition 1.1. For $\Re(s)>0$, The lower incomplete gamma function is defined as:

$$
\gamma(s, x)=\int_{0}^{x} t^{s-1} e^{-t} d t
$$

and the upper incomplete gamma function is defined as:

$$
\Gamma(s, x)=\int_{x}^{\infty} t^{s-1} e^{-t} \mathrm{~d} t .
$$

Clearly,

$$
\Gamma(s, z)=\Gamma(s)-\gamma(s, z) .
$$

Moreover, $\gamma(s, x) \longrightarrow \Gamma(s)$ as $x \longrightarrow \infty$ and $\Gamma(s, 0)=\Gamma(s)$.

Definition 1.2. The incomplete beta function, a generalization of the beta function, is defined as follows: for $0 \leq x \leq 1, \Re(s)>0$ and $\Re(t)>0$

$$
\beta(s, t ; x)=\int_{0}^{x} u^{s-1}(1-u)^{t-1} d u .
$$

Clearly,

Proposition 1.1. For $0 \leq x \leq 1, \Re(s)>0$ and $\Re(t)>0$

1. $\beta(1,1, x)=x$,

Received : 05-February-2016, Accepted : 05-May-2016 
2. $\beta(s, t, 1)=\beta(s, t)$,

3. $\beta(1, t, x)=\frac{1-(1-x)^{t}}{t}$,

4. $\beta(s, 1, x)=\frac{x^{s}}{s}$.

The following properties are needed:

Proposition 1.2. [5] For $\Re(s)>0$

1. $\Gamma(1, x)=e^{-x}$,

2. $\Gamma\left(\frac{1}{2}, x\right)=\sqrt{\pi} \operatorname{erfc}(\sqrt{x})$,

3. $\gamma(1, x)=1-e^{-x}$,

4. $\gamma\left(\frac{1}{2}, x\right)=\sqrt{\pi} \operatorname{erf}(\sqrt{x})$.

5. $\beta\left(\frac{1}{2}, \frac{1}{2}, x\right)=2 \arcsin (\sqrt{x})$.

The incomplete gamma functions are used in the discussions of power-law relaxation times in complex physical systems (see([13])); logarithmic oscillations in relaxation times for proteins (see ([9])); Gaussian orbital and exponential (Slater) orbital in quantum chemistry (see ([11]) and ([12])). The properties of these functions and other related functions are listed in many references( see [1], [2], [3], , [6], [7] , [8], [10] [14] and [15]). Recently, the products of incomplete gamma functions are represented in integrals which are given in polar coordinates ( see [4])

\section{Main Results}

Using the substitutions $u=z w$ and $v=z(1-w)$ one can give get the following

$$
\begin{aligned}
\Gamma(a) \Gamma(b) & =\int_{0}^{\infty} \int_{0}^{\infty} e^{-u} u^{a-1} e^{-v} v^{b-1} d v d u \\
& =\int_{0}^{1} \int_{0}^{\infty} e^{-z w}(z w)^{a-1} e^{-z(1-w)}(z(1-w))^{b-1}(z d z d w) \\
& =\gamma(a+b) \beta(a, b) .
\end{aligned}
$$

This proves the product $\Gamma(a) \Gamma(b)$ equals $\gamma(a+b) \beta(a, b)$.The interesting question: Following a similar proof, does the product of incomplete gamma functions satisfy a similar proposition. The answer is given in the following theorem

Theorem 2.1. For $t>0, \Re(a)>0$ and $\Re(b)>0$. The product $\gamma(a, t) \gamma(b, s)$ satisfies

$$
\begin{aligned}
\gamma(a, t) \gamma(b, s) & =\int_{0}^{\frac{t}{s+t}} \gamma\left(a+b, \frac{s}{1-w}\right) w^{a-1}(1-w)^{b-1} d w \\
& +\int_{\frac{t}{s+t}}^{1} \gamma\left(a+b, \frac{t}{w}\right) w^{a-1}(1-w)^{b-1} d w .
\end{aligned}
$$

Proof. Using the substitutions $u=z w$ and $v=z(1-w)$ along with Definition 1.1, we get

$$
\begin{aligned}
\gamma(a, t) \gamma(b, s) & =\int_{0}^{t} \int_{0}^{s} e^{-u} u^{a-1} e^{-v} v^{b-1} d v d u \\
& =\int_{0}^{\frac{t}{s+t}} \int_{0}^{\frac{s}{1-w}} e^{-z w}(z w)^{a-1} e^{-z(1-w)}(z(1-w))^{b-1}(z d z d w) \\
& +\int_{\frac{t}{s+t}}^{1} \int_{0}^{\frac{t}{w}} e^{-z w}(z w)^{a-1} e^{-z(1-w)}(z(1-w))^{b-1}(z d z d w) \\
& =\int_{0}^{\frac{t}{s+t}} \gamma\left(a+b, \frac{s}{1-w}\right) w^{a-1}(1-w)^{b-1} d w \\
& +\int_{\frac{t}{s+t}}^{1} \gamma\left(a+b, \frac{t}{w}\right) w^{a-1}(1-w)^{b-1} d w .
\end{aligned}
$$


letting $s \longrightarrow \infty$ in (2.1), then we get

Corollary 2.1. For $t>0, \Re(a)>0$ and $\Re(b)>0$

$$
\gamma(a, t) \Gamma(b)=\int_{0}^{1} \gamma\left(a+b, \frac{t}{w}\right) w^{a-1}(1-w)^{b-1} d w=\int_{0}^{1} \gamma\left(a+b, \frac{t}{1-w}\right)(1-w)^{a-1} w^{b-1} d w .
$$

Also,

$$
\Gamma(a, t) \Gamma(b)=\int_{0}^{1} \Gamma\left(a+b, \frac{t}{w}\right) w^{a-1}(1-w)^{b-1} d w=\int_{0}^{1} \Gamma\left(a+b, \frac{t}{1-w}\right)(1-w)^{a-1} w^{b-1} d w .
$$

Now, the following is an extension of the well-known result that $\Gamma(a) \Gamma(1-a)=\frac{\pi}{\sin (\pi x)}$

Corollary 2.2. For $0<\Re(a)<1$ and $x>0$

$$
\Gamma(a, x) \Gamma(1-a)=\int_{0}^{1} \frac{e^{-\frac{x}{w}}}{w^{1-a}(1-w)^{a}} d w=\int_{0}^{1} \frac{e^{-\frac{x}{1-w}}}{(1-w)^{1-a} w^{a}} d w .
$$

Moreover, If $f$ has Laplace transform then

$$
\int_{0}^{\infty} f(x) \Gamma(a, x) d x=\frac{1}{\Gamma(1-a)} \int_{0}^{1} \frac{F\left(\frac{1}{w}\right)}{w^{1-a}(1-w)^{a}} d w=\frac{1}{\Gamma(1-a)} \int_{0}^{1} \frac{F\left(\frac{1}{1-w}\right)}{(1-w)^{1-a} w^{a}} d w .
$$

Proof. Use Proposition 1.2 and (2.3) with $b=1-a$. Now, if $f$ has Laplace transform $F$ it satisfies that

$$
\int_{0}^{\infty} e^{-\frac{x}{w}} f(x) d x=F\left(\frac{1}{w}\right) .
$$

Therefore,

$$
\begin{aligned}
\int_{0}^{\infty} f(x) \Gamma(a, x) d x & =\frac{1}{\Gamma(1-a)} \int_{0}^{\infty} \int_{0}^{1} \frac{e^{-\frac{x}{w}} f(x)}{w^{1-a}(1-w)^{a}} d w d x \\
& =\frac{1}{\Gamma(1-a)} \int_{0}^{1} \frac{1}{w^{1-a}(1-w)^{a}} \int_{0}^{\infty} e^{-\frac{x}{w}} f(x) d x d w \\
& =\frac{1}{\Gamma(1-a)} \int_{0}^{1} \frac{F\left(\frac{1}{w}\right)}{w^{1-a}(1-w)^{a}} d w=\frac{1}{\Gamma(1-a)} \int_{0}^{1} \frac{F\left(\frac{1}{1-w}\right)}{(1-w)^{1-a} w^{a}} d w
\end{aligned}
$$

Corollary 2.3. For $x>0$

$$
\operatorname{erfc}(\sqrt{x})=\frac{1}{\pi} \int_{0}^{1} \frac{e^{-\frac{x}{1-w}}}{\sqrt{w(1-w)}} d w=\frac{1}{\pi} \int_{0}^{1} \frac{e^{-\frac{x}{w}}}{\sqrt{w(1-w)}} d w .
$$

Moreover, If $f$ has Laplace transform then

$$
\int_{0}^{\infty} f(x) \operatorname{erfc}(\sqrt{x}) d x=\frac{1}{\pi} \int_{0}^{1} \frac{F\left(\frac{1}{1-w}\right)}{\sqrt{w(1-w)}} d w=\frac{1}{\pi} \int_{0}^{1} \frac{F\left(\frac{1}{w}\right)}{\sqrt{w(1-w)}} d w .
$$

Proof. Use Proposition 1.2 and Corollary 2.2 with $a=\frac{1}{2}$.

Using Corollary 2.2 with $f(x)=e^{-b x}, b>0$ we get

Proposition 2.1. For $b>0$

$$
\int_{0}^{1} \frac{1}{1+b w}\left(\frac{w}{1-w}\right)^{a} d w=\frac{\pi \csc (\pi a)}{b}\left(1-\frac{1}{(1+b)^{a}}\right)
$$

For example,

$$
\int_{0}^{1} \frac{1}{1+15 w}\left(\frac{w}{1-w}\right)^{1 / 4} d w=\frac{\pi}{15 \sqrt{2}}
$$


Using (2.3) we have

Example 2.1. For $a>0$

$$
\int_{0}^{1} \frac{e^{-\frac{a}{w}}}{\sqrt{w-w^{2}}} d w=\pi \operatorname{erfc}(\sqrt{a})
$$

Example 2.2. For $\Re(b)>0$ and $\Re(a+b)>0$

$$
\begin{aligned}
\int_{0}^{\infty} x^{b-1} \Gamma(a, x) d x & =\frac{1}{\Gamma(1-a)} \int_{0}^{1} \frac{\Gamma(b) w^{b}}{w^{1-a}(1-w)^{a}} d w \\
& =\frac{\Gamma(b)}{\Gamma(1-a)} \int_{0}^{1} w^{b+a-1}(1-w)^{-a} d w \\
& =\frac{\Gamma(b)}{\Gamma(1-a)} \beta(a+b, 1-a)=\frac{\Gamma(a+b)}{a} .
\end{aligned}
$$

Theorem 2.2. For $\Re(a)>0$ and $\Re(b)>0$

$$
\gamma(a, t) \gamma(b, s)=\beta(a, b) \Gamma(a+b)-\int_{s}^{s+t} \beta\left(a, b, 1-\frac{s}{z}\right) e^{-z} z^{a+b-1} d z+\int_{t}^{s+t} \beta\left(a, b, \frac{t}{z}\right) e^{-z} z^{a+b-1} d z .
$$

Proof. Without loss of generality we assume $t<s$, interchanging $t$ and $s$ gives the same result. Using the substitutions $u=z w$ and $v=z(1-w)$ along with Definition 1.1, we get

$$
\begin{aligned}
\gamma(a, t) \gamma(b, s) & =\int_{0}^{t} \int_{0}^{s} e^{-u} u^{a-1} e^{-v} v^{b-1} d v d u \\
& =\int_{0}^{t} \int_{0}^{1} e^{-z w}(z w)^{a-1} e^{-z(1-w)}(z(1-w))^{b-1}(z d w d z) \\
& +\int_{t}^{s} \int_{0}^{\frac{t}{z}} e^{-z w}(z w)^{a-1} e^{-z(1-w)}(z(1-w))^{b-1}(z d w d z) \\
& +\int_{s}^{s+t} \int_{1-\frac{s}{z}}^{\frac{t}{z}} e^{-z w}(z w)^{a-1} e^{-z(1-w)}(z(1-w))^{b-1}(z d w d z) \\
& =\gamma(a+b, t) \beta(a, b)+\int_{t}^{s} z^{a+b-1} e^{-z} \beta\left(a, b, \frac{t}{z}\right) d z+\int_{s}^{s+t} z^{a+b-1} e^{-z} \beta\left(a, b, \frac{t}{z}\right) d z \\
& -\int_{s}^{s+t} z^{a+b-1} e^{-z} \beta\left(a, b, 1-\frac{s}{z}\right) d z \\
& =\beta(a, b) \Gamma(a+b)-\int_{s}^{s+t} \beta\left(a, b, 1-\frac{s}{z}\right) e^{-z} z^{a+b-1} d z+\int_{t}^{s+t} \beta\left(a, b, \frac{t}{z}\right) e^{-z} z^{a+b-1} d z .
\end{aligned}
$$

Corollary 2.4. For $\Re(a)>0$ and $\Re(b)>0$

$$
\gamma(a, t) \Gamma(b)=\beta(a, b) \Gamma(a+b)-\int_{t}^{\infty} \beta\left(a, b, 1-\frac{t}{z}\right) e^{-z} z^{a+b-1} d z .
$$

Equation 2.4, Proposition 1.2 and Proposition 1.1 give the following result

Corollary 2.5. $e^{-t} \Gamma(b)=\int_{t}^{\infty}\left(\frac{z^{b}-t^{b}}{b}\right) e^{-z} d z=\frac{\Gamma(b+1, t)-t^{b} e^{-t}}{b}$.

Proof. take $a=1$ into (2.4), then apply Proposition 1.1.

Corollary 2.6. For $\Re(b)>0$ and $t>0$

$$
\operatorname{erfc}(\sqrt{t})=\frac{1}{\sqrt{\pi} \Gamma(b)} \int_{t}^{\infty} \beta\left(\frac{1}{2}, b, 1-\frac{t}{z}\right) e^{-z} z^{b-\frac{1}{2}} d z .
$$

In particular,

$$
\operatorname{erfc}(\sqrt{t})=\frac{2}{\pi} \int_{t}^{\infty} \arccos \left(\sqrt{\frac{t}{z}}\right) e^{-z} d z=\frac{2}{\pi} \int_{t}^{\infty} \arcsin \left(\sqrt{1-\frac{t}{z}}\right) e^{-z} d z
$$


Proof. Take $a=\frac{1}{2}$ into (2.4), then apply Proposition 1.1 to prove (2.9). For (2.10), use (2.9) with $b=\frac{1}{2}$ and use the identity that for $0 \leq x \leq 1, \arcsin (\sqrt{x})=\arccos (\sqrt{1-x})$.

Example 2.3. Using 2.10 with $t=1$

$$
\int_{1}^{\infty} \operatorname{arcsec}(\sqrt{z}) e^{-z} d z=\frac{\pi}{2} \operatorname{erfc}(1)
$$

\section{References}

[1] Agarwal, P. and Nieto, J. J., Some fractional integral formulas for the Mittag-Leffer type function with four parameters. Open Mathematics 13 (2015), no. 1, 537-546.

[2] Agarwal, P., Chand, M. and Jain, S., Certain integrals involving generalized Mittag-Leffer functions. Proc. Natl. Acad. Sci., India, Sect. A Phys. Sci. 85 (2015), no. 3, 359-371.

[3] Agarwal, P. and Jain, S., A new class of integral relations involving a general class of polynomials and I-functions. Walailak J Sci \& Tech. 12 (2015), no. 11, 1009-1018.

[4] AlAhmad, R., Products of incomplete gamma functions. Analysis ISSN (Online) 2196-6753, ISSN (Print) 01744747, DOI: 10.1515/anly-2015-0012 (2015).

[5] Apelblat, A., Table of definite and infinite integrals. Physical Sciences Data, Elsevier Scientific Publishing Co., Amsterdam, 1983.

[6] Çetinkaya, A., The incomplete second Appell hypergeometric functions. Applied Mathematics and Computation 219 (2013), 8332-8337.

[7] Choi, J. and Agarwal, P., Certain integral transforms for the incomplete functions.Appl. Math. 9 (2015), no. 4, 2161-2167.

[8] Gradshteyn ,I. S. and Ryzhik ,I. M., Tables of integrals, series and products. Academic Press, New York, 1980.

[9] Metzler, R., Klafter, J. and Jortner, J., Hierarchies and logarithmic oscillations in the temporal relaxation patterns of proteins and other complex systems. Proc. Nat. Acad. Sci. 96 (1999), no. 20, 11085-11089.

[10] Miller, A. R. and Moskowitz, I. S., On certain generalized incomplete gamma functions. J. Comp. Appl. Math. 91 (1963), no. 2, 179-190.

[11] Shavitt, S., The gaussian function in calculations of statistical mechanics and quantum mechanics, chapter in methods in computational physics. Academic Press, New York, 1963.

[12] Shavitt, I. and Karplus, M., Gaussian-transform method for molecular integrals. I. Formulation for energy integrals. J. Chem. Phys. 43 (1965), no. 2, 398-414.

[13] Sornette, D., Multiplicative processes and power laws. Phys. Rev. E. 57 (1998), no. 4, 4811-4813.

[14] Srivastava, H. M. and Agarwal, P., Certain fractional integral operators and the generalized incomplete hypergeometric functions. Applications and Applied Mathematics, 2013.

[15] Temme, N., Uniform asymptotic expansions of the incomplete gamma functions and the incomplete beta function. Math. Comp. 29 (1975), no. 132, 1109-1114.

\section{Affiliations}

RAMI ALAHMAD

AdDRESS: Yarmouk University, Dept. of Mathematics, 21163, Irbid-Jordan.

E-MAIL: rami_thenat@yu.edu.jo 